\title{
Glaucoma screening by means of an optic disc grid
}

\author{
R. A. HITCHINGS, D. B. BROWN, AND S. A. ANDERTON \\ From the Glaucoma Unit, Moorfields Eye Hospital, High Holborn, London WC1V 7AN
}

SUMMARY With a superimposed optic disc grid a study was performed to see whether visual field defects could be identified solely by changes visible on the neuroretinal rim. It was shown that a nonmedically qualified observer could with fair accuracy correctly identify patients with and without glaucomatous visual field defects. The sources of error are discussed.

Glaucoma screening depends at present on the findings of tonometry, visual field testing, and examination of the optic discs. The results obtained by mass tonometry set a standard against which the results of the other 2 tests must be compared. With 21 $\mathrm{mmHg}$ as the arbitrary upper limit of normal approximately $40 \%$ of patients with chronic glaucoma will have an intraocular pressure below this level at the time of screening, while only $10 \%$ of those with pressures greater than $21 \mathrm{mmHg}$ have definite glaucoma at the time of screening. ${ }^{1}$ Visual field testing runs into similar problems with false positives and false negatives. Unless an elaborate learning programme for the patients is embarked upon, the false positive results from an initial test may rise as high as $50 \%$, while the false negative rate has not been accurately identified on screening studies. ${ }^{1}$

Optic disc screening also presents problems. Reliance on pallor of the optic disc may be misleading, as many optic discs appear unnaturally pink owing to colour leak from the peripapillary choroid or from coexisting nuclear sclerosis. ${ }^{2}$ Biomicroscopic examination by a skilled observer is needed to identify focal pallor of the neuroretinal rim. A high proportion of significant field defects may be correctly identified from examination of stereo photographs when this examination is performed by an experienced observer. ${ }^{3}$ Changes in the colour of the neuroretinal rim have been used in conjunction with a grid system in glaucoma screening programmes ${ }^{4}$ and have been shown to be of use in the identification of visual field defects. This system, however, was complex and relied on colour rather than topographic changes.

It seemed worthwhile to produce a simple optic disc grid system to see whether this system would achieve the same results. The optic disc grid $^{5}$ was used

Correspondence to $\mathrm{Mr} \mathrm{R}$. A. Hitchings. in the study to see whether a nonmedically trained individual could correctly identify patients having visual field defects from a group of patients attending the Glaucoma Unit at Moorfields Eye Hospital, High Holborn, London.

\section{Material and methods}

Optic disc photographs giving a stereo effect of 102 patients known to be attending the Glaucoma Unit but for whom no other clinical details were available were studied by 3 observers. The first observers (D.B.B. and R.A.H.) selected paired photographs of the optic disc from the files of the Glaucoma Unit, Moorfields Eye Hospital, High Holborn. The photo-

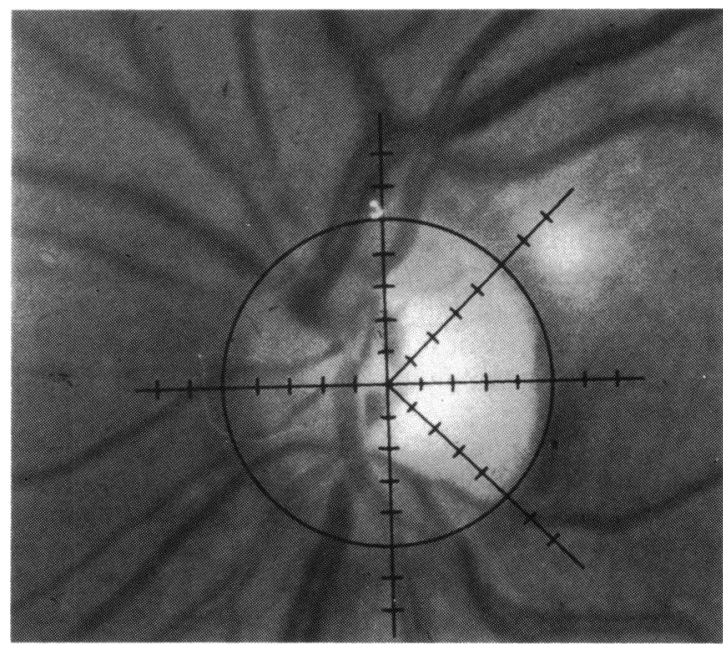

Fig. 1 Optic disc photographed with superimposed optic disc grid. In this case the neuroretinal rim is greater than one division in thickness. 


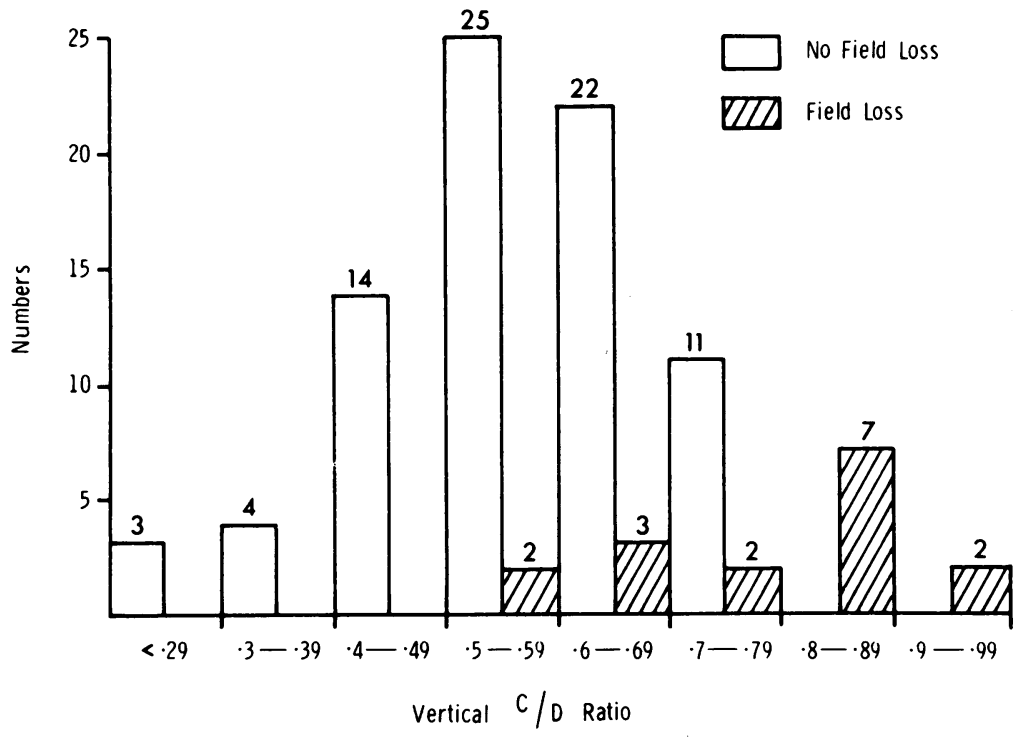

Fig. 2 Histogram showing study sample. A: Vertical C/D ratio. B: Horizontal C/D ratio.

Fig. 2A

graphs chosen were in good focus and gave a stereo effect. No true stereo photographs were available. Those patients whose 'stereo' photographs coincided with the obtaining of a 3 isoptre kinetic visual field from a Goldmann perimeter taken within 4 months of the photographs were chosen for this study. In this way 'stereo' photographs from 102 patients' eyes were selected.

A note was made of those visual fields with a 'nerve fibre bundle' type of visual field defect (of minimum size $10^{\circ}$ in diameter involving one or more isoptre). These patients were considered to have 'visual field defects.' Patients with no demonstrable abnormality in the visual field as well as those whose only change in the visual field was peripheral constriction of 'baring of the blind spot' were considered to have 'normal fields.'

Neuroretinal rim thickness on the stereo disc photographs was checked against the occurrence of visual field defects. The optic disc grid ${ }^{5}$ (Fig. 1) was used to measure the rim thickness. As a result a figure of just below one division or less on the optic disc grid was found to coincide with the occurrence of 'visual field defects' as described above.

The third observer (S.A.A.), a glaucoma technician, was instructed to measure rim thickness on the 102 stereo disc pairs. She was told to identify those eyes with an area of neuroretinal rim less than one division. on the optic disc grid in thickness. No further information was given. The results obtained by this third observer were compared with the known visual field and optic disc appearance noted by the first 2 observers.

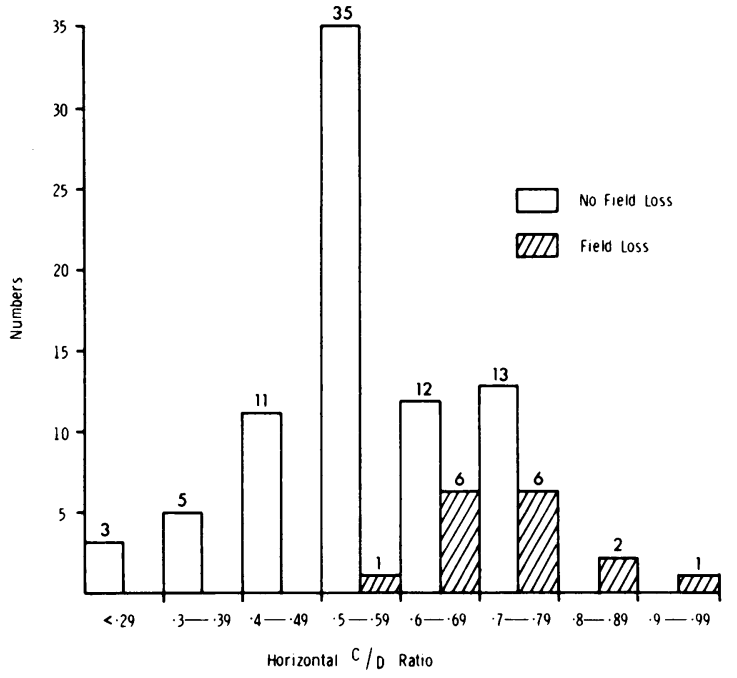

Fig. 2B

\section{Results}

The study sample consisted of 102 stereo disc pairs. The vertical and horizontal (cap/disc) (C/D) ratio in this study sample has been set out in Fig. 2, from which it will be seen that the majority had a C/D ratio ranging from 0.4 to $0 \cdot 7$.

The results obtained from optic disc screening are as follows. Of 16 eyes with 'visual field defects' 12 were correctly diagnosed. Of 86 eyes with 'normal visual fields' 76 were correctly diagnosed. This gave a sensitivity of $75 \%$ and a specificity of $86 \%$.

There were 4 false negatives: in one case a small 
notch was missed; in 2 cases there were small and normal looking discs.

There were 10 false positives: 3 with thin rims, positive according to our criteria; 3 with large discs and large cups each with a rim at one division in width; 2 with indefinite rims because of the presence of a sloping cup wall; 2 with a horizontal cup together with a thin rim existing at the horizontal meridian alone.

A comparison of the $C / D$ ratios in eyes with and without field defects is also set out in the Fig. 2.

\section{Discussion}

In previous papers ${ }^{36}$ it was shown that a good correlation between the appearance of the neuroretinal rim and the existence of a visual field defect (noted on kinetic bowl perimetry). More recently Shiose ${ }^{7}$ noted that changes at the neuroretinal rim suggesting glaucomatous field loss could be identified by means of a 'glaucoma disc pattern viewer'. He simultaneously projected the disc pattern viewer on to a projected image of a fundus photograph for the identification of glaucomatous discs.

In this paper we have shown that a simple disc grid placed on one of a stereo disc pair viewed with a simple viewer against a cold light illuminator allowed recognition of 'nerve fibre bundle type' of visual field defects in a high proportion of cases, giving a sensitivity of $75 \%$ and a specificity of $86 \%$. More importantly, this recognition was achieved by a glaucoma technician asked to identify thickness of the neuroretinal rim alone when screening these optic discs. This nonmedically qualified observer could separate those optic discs with visual field loss from those without in the majority of the optic discs studied.

There were 3 major sources of error. Firstly, errors occurred from a failure to observe parts of the neuroretinal rim narrow enough to qualify for field loss by the criteria selected. Observers 1 and 2 were able to correctly identify one such case. It was felt that greater magnification and further practice should certainly reduce the chances of this error occurring. Occasional cases of optic disc with a narrow rim restricted to the horizontal meridian (which may be seen in a small number of 'normal' eyes) were also included by our criteria as patients with possible field loss. However, recognition of this as a normal variant would remove its appearance among a list of false positives.

Secondly, errors were caused by difficulty in assessing the junction between the cup wall and the neuroretinal rim. A review of the 10 false positives showed that 2 such optic discs could be so classified. We believe that improved magnification would go some way to reducing this source of error.
Thirdly, and perhaps most importantly, was the problem posed by the large optic cup. Eyes having large optic discs tend to have a large optic cup. The optic disc would be of sufficient size to ensure that the surrounding rim was thin enough to be positive by our criteria. Three such false positives were identified in this series. Greater accuracy may be achieved by incorporating a cut-off point for overall cup size suggestive of glaucoma as well as by using criteria for minimum rim thickness.

It is worth looking at the relationship of the C/D ratio and the neuroretinal rim thickness to visual field loss. Fig. 2 shows the considerable overlap in C/D ratios seen in the patients with and without visual field defects in this study. This overlap suggests that for those optic discs with a $C / D$ ratio of less than 0.8 an additional parameter must be chosen when looking for visual field loss. Those optic discs with a $\mathrm{C} / \mathrm{D}$ ratio of $>0.8$ were all read as positive by the grid system. It is of interest that Gloster ${ }^{8}$ noted a dramatic increase in the likelihood of glaucomatous visual fields defects being present when the $C / D$ ratio changed from 0.7 to 0.8 (from 2:1 to 20:1). Analysis of the false positives and false negatives in the $C / D$ ratio in the range $0.5-0.8$ showed that neuroretinal rim thickness rather than C/D ratio may be a more accurate method of assessing visual field loss in this type of patient. For screening purposes, however, perhaps both the existence of a $C / D$ ratio $>0.8$ as well as selective areas of rim thickness less than one division on the optic disc grid should be chosen.

Glaucoma screening by means of an optic disc grid has been recently and elegantly discussed by Shiose $e t$ $a l .{ }^{4}$ Their system involved superimposition of a projected disc grid on a photograph of the optic disc. They used a monocular photograph and identified areas of centrepetal extension of the central pallor of the optic disc. Although with an earlier study Shiose ${ }^{7}$ reported sensitivity and specificity of 89 and $93 \%$ respectively (values comparing well with the sensitivity and specificity values obtained from identification of visual field loss by means of stereo disc photographs ${ }^{3}$ ), the results he obtained from monocular photographs when screening were disappointing. For out of 102 eyes with extended pallor (signifying loss of visual field) identified in his screening series only 52 were found to have a visual field defect, while a further 4 eyes with a visual field defect did not have such extension of pallor. Although fewer false positives were seen, should a retinal nerve fibre bundle defect coexist, this defect was not seen frequently enough for his system to be relied on solely for screening purposes. It remains to be seen whether the transfer of selective stereo photographs from the pilot study reported here into a screening study will achieve the same degree of sensitivity and specificity. 


\section{CONCLUSION}

This study has shown that paramedical personnel can with fair accuracy identify by means of an optic disc grid the presence or absence of glaucomatous visual field defects. Such a system could be made use of in screening for glaucoma, especially when allied to a method for measuring overall C/D ratios of $>0 \cdot 8$. Some errors in this system exist as well as methods by which the accuracy could be improved.

We thank Mrs Kay Mills for typing the manuscript.

\section{References}

1 Hitchings RA. Glaucoma screening. Res Clin Forums 1981; 3: 109-14.
2 Gloster J, Parry DG. Use of photographs for measuring cupping of the optic disc. Br J Ophthalmol 1974; 58: 850-62.

3 Hitchings RA, Spaeth GL. The optic disc in glaucoma. II. Correlation of the appearance of the optic disc with the visual field. Br J Ophthalmol 1977; 61: 107-13.

4 Shiose Y, Komiro Km Itch T, Amano M, Kawase Y. New system for mass screening of glaucoma as part of automated multiphasic health testing services. Jpn J Ophthalmol 1981; 25: 160-77.

5 Hitchings RA, Genio C, Anderton S, Clark P. An optic disc grid. Its evaluation in reproducibility studies on the cup/disc ratio. $\mathrm{Br} J$ . Ophthalmol 1983; 67: 356-61.

6 Hoskins HD, Gelber EC. Optic disc topography and visual field defects in patients with increased intraocular pressure. Am J Ophthalmol 1975; 80: 284-90.

7 Shiose Y. Quantitative disc patterns as a new parameter for glaucoma screening. Glaucoma 1979; 1: 41-9.

8 Gloster J. A quantitative relationship between cupping of the optic disc and visual field loss in chronic simple glaucoma. $\mathrm{Br} \mathrm{J}$ Ophthalmol 1978; 62: 665-9. 